\title{
Le repas de noces dans la Crète de la Vénétocratie
}

Nouvelles informations sur l'histoire de la nourriture à Candie au $\mathrm{XVII}^{\mathrm{e}}$ siècle

The Wedding Feast in Crete under Venetian Rule: New Information on the History of Food in 17th Century Candia

\section{Georgia K. Varzelioti}

\section{(2) OpenEdition}

\section{Journals}

Édition électronique

URL : https://journals.openedition.org/ceb/7684

DOI : $10.4000 /$ ceb.7684

ISSN : 2261-4184

\section{Éditeur}

INALCO

Édition imprimée

ISBN : 978-2-85837-230-6

ISSN : 0290-7402

\section{Référence électronique}

Georgia K. Varzelioti, « Le repas de noces dans la Crète de la Vénétocratie », Cahiers balkaniques [En ligne], Hors-série | 2016, mis en ligne le 16 mars 2017, consulté le 06 juillet 2021. URL : http:// journals.openedition.org/ceb/7684 ; DOI : https://doi.org/10.4000/ceb.7684

Ce document a été généré automatiquement le 6 juillet 2021.

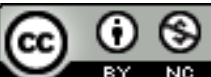

Cahiers balkaniques est mis à disposition selon les termes de la Licence Creative Commons Attribution - Pas d'Utilisation Commerciale 4.0 International. 


\title{
Le repas de noces dans la Crète de la Vénétocratie
}

\author{
Nouvelles informations sur l'histoire de la nourriture à Candie au \\ XVII ${ }^{\mathrm{e}}$ siècle \\ The Wedding Feast in Crete under Venetian Rule: New Information on the \\ History of Food in 17th Century Candia
}

Georgia K. Varzelioti

1 Pendant les dernières décennies, médecins, nutritionnistes et historiens de la nutrition s'accordent à reconnaître les bienfaits incontestables du régime méditerranéen et en particulier de la cuisine crétoise, d'autant plus que ce mode alimentaire, qui repose sur une variété d'aliments ainsi que sur un mode traditionnel de préparation, est associé à la longévité et à une meilleure qualité de vie (PSILAKI \& PSILAKIS, 1999), (RENAUD, 2004). Les habitudes culinaires des Crétois pendant les quatre siècles de la Vénétocratie, établies dans le cadre de la cohabitation créto-vénitienne et dans un contexte d'interactions socioculturelles plus larges, présentent un intérêt particulier. L'échange entre tradition byzantine et culture des colons, se voit couronné par la création d'une société crétovénitienne mixte, et par un phénomène culturel unique, ayant de nombreuses applications dans l'art, la littérature, le théâtre, la mode et l'architecture entre autres (MAltezou, 1988, 107-159), (MAltezou, 1998, 3-20), (MALTEzou, 2008, 21-57)1․

2 C'est justement dans ce contexte historique que s'inscrit le mode culinaire sur l'île, lié non seulement à la survie, mais aussi au bien-être - fêtes familiales, populaires et religieuses, jubilées, foires. Plusieurs questions émergent donc: que se passait-il en campagne et dans les centres urbains ? Y avait-il des différences marquantes entre la culture alimentaire de l'élite et celle du peuple? Quel était le coût des produits alimentaires et que valaient les articles d'usage ménager, c'est-à-dire le matériel de cuisine et de service? Quelles étaient les origines gastronomiques byzantines et en quoi consistaient les influences vénitiennes? Les réponses abondent dans la bibliographie relative à ce thème, mais aussi dans de nombreuses sources manuscrites disponibles, telles que les documents administratifs et notariés, les récits de voyages ou les textes 
littéraires, qui nous permettent de dégager les grandes tendances alimentaires de l'époque (ANAGNOSTAKIS, 2013, 175-181), (KALAMARA, 2010, 8-17), (KISLINGER, 1999, 194-206), (KoDER, 2005, 19-26), (MOTSIAS, 1998), (PANOPOULOU, 1998, 465-495), (PAPADAKIS, 1978), (PSILAKI \& PSILAKIS, 1999), (PSILAKIS, 1998, 319-329), (RORATO, 2003), (TEYSSEYRE, 1999), (VLASSI, 1998, 55-75, avec toute la bibliographie précédente), (VLASSI, 2010, 366-373).

Cet article apportera quelques éléments complémentaires sur l'histoire culinaire crétoise au cours de la domination vénitienne, et plus particulièrement sur l'un des moments les plus heureux de la vie privée: le festin des noces, un événement réjouissant, omniprésent dans des études historiques, ethnologiques et littéraires (PAPADAKIS, 1980 ; VARZELIOTI, 2011, 63-82), avec la documentation et la bibliographie antérieure). Les comédies crétoises de la Renaissance nous fournissent également de précieuses indications sur ce sujet (VLASSI, 1998, 55-75). On pourrait se référer à titre d'exemple à la scène des domestiques constamment affamés qui cherchent à assouvir leur faim et dont le point culminant se trouve dans la description du banquet de mariage qui accompagne le dénouement heureux: le mariage du couple amoureux (VARZELIOTI, 2011, 81-82). Cette image s'enrichit de nouvelles informations fournies par une source inestimable, le libro dei conti (le livre de comptes) d'une riche veuve candiote, que j'ai repéré, il y a quelques années, dans les Archives d'État de Venise, lors de mes recherches en tant que boursière de l'Institut hellénique d'études byzantines et post-byzantines de la ville (VARZELIOTI, 2010, 311-318).

4 Venons-en maintenant à cette source. En 1609, une femme, appartenant à la noblesse de Candie, Pandoussa Mezeri, veuve du médecin-philosophe de la ville, Victor Mezeri, a commencé à enregistrer les dépenses relatives à la gestion du patrimoine familial. Le texte copié a été sauvegardé dans le registre du notaire de Candie Giacomo Cortesan, attaché à un document, rédigé pour une affaire judiciaire de la veuve. Il est écrit à la première personne, sous forme de journal, en langue grecque en caractères latins, s'étend sur cinquante-trois pages, et recouvre la période allant de 1609 à 1620. Pendant ce temps, Mezeri a marié ses cinq filles. Les dépenses pour ces célébrations de mariage, joyeuses, mais coûteuses, ont été enregistrées en détail par la mère et reflètent les coutumes et les rites de mariage de la noblesse en Crète, pendant la première moitié du $\mathrm{XVII}^{\mathrm{e}}$ siècle. La consultation de ces registres évoque, de manière éloquente, l'ambiance festive qui régnait à la maison lors de la préparation et le jour même des noces.

\section{La richesse du menu}

5 Selon le registre établi par la Mezeri, la célébration débutait quelques jours avant la cérémonie. La maison s'ouvrait pour accueillir, en grande pompe, le fiancé, sa famille, les parents et les amis. Dans le cadre des préparatifs, on faisait appel à de nombreux professionnels : des musiciens pour égayer l'atmosphère, un professeur de danse pour

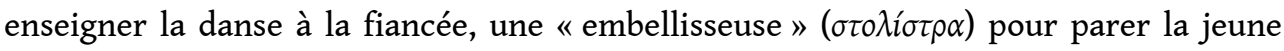
fille pendant le séjour du fiancé à la maison, et bien évidemment le jour des noces. On engageait aussi des serveurs et des cuisiniers pour la préparation des mets et surtout celle de la table de cérémonie. Les festins de noces des cinq filles témoignent d'une surabondance de vin et des mets copieux : viande, volaille, gibier à plumes, charcuterie, fruits, légumes, riz et pâtes, ainsi que des friandises traditionnelles : des bonbons dorés $(\chi \rho \circ v \sigma \omega \mu \varepsilon \dot{v} \alpha \alpha \alpha \chi \alpha \rho \omega \tau \tau \alpha)$, des douceurs arrosées de l'eau de rose, des tourtes, des confeta 
(des amandes enrobées de sucre) et le point d'honneur, les xerotigana, une sorte de beignets arrosés de miel et de noix concassées (VARZELIOTI 2011, 78-81).

Voyons maintenant, à titre d'exemple, les denrées utilisées pour la préparation du festin qui a eu lieu à la demeure Mezeri, et plus précisément celui pour les noces de Marietta Mezeropoula avec le gentilhomme Michele Vidali, en 1617. Selon le registre de la veuve Mezeri, ont été achetés : douze canetons, vingt pigeonneaux, quatre agneaux, des grives, des canetons sauvages et d'autres volatiles, deux jambons, du fromage, deux poules couveuses avec des œufs, quatre $\sigma \alpha \lambda \tau \imath \tau \sigma o u ́ v i \alpha$ (c'est l'appellation d'un genre de saucisse dans le dialecte créto-vénitien) (VLASSI, 1998, 70), huit livres de riz, du lait, huit choux, dix bottes de céleri, des radis, des raisins, des citrons et des oranges amères, du lard, dix livres de sucre pour les pâtisseries de toute sorte, de la cannelle, du poivre, des clous de girofle, deux boîtes d'épices, des prunes, des mirabelles, de l'eau de rose, des châtaignes, des graines de fenouil, du raisin de Corinthe, des amandes, du miel, des pommes, des coings, des noix de pin pour les tourtes, du vin muscat, du vin marouva et soixante-dix œufs (VARZELIOTI, 2011, 80-81)2.

7 Cette liste nous conduit à exprimer certaines réflexions. Elle témoigne d'une profusion de produits, locaux et importés. Il est clair que nous sommes assez loin des habitudes culinaires des couches sociales inférieures, dont l'alimentation ordinaire, à l'exception du menu d'un repas de fête, est peu carnée et essentiellement constituée d'aliments d'origine végétale (légumineuses et légumes). En revanche, dans les milieux aisés, l'alimentation est très carnée : elle repose sur la consommation des viandes, du gibier et de la charcuterie (PSILAKIS, 1998, 320-329).

\section{Abondance, diversité et influences culinaires}

8 Ce qui est vraiment impressionnant c'est l'abondance et la diversité des plats, étroitement liées au banquet de noces et à la solennité du jour, aussi bien dans la sphère privée que sociale. Il est évident que le faste de cette réception ne correspond pas au mode alimentaire quotidien des seigneurs ou bourgeois prospères. En effet, les denrées sont les mêmes, mais c'est la procession quantitative et qualitative de mets qui fait la différence. C'est que la splendeur de la célébration est signe de distinction qui atteste à la fois du statut social et financier des jeunes mariés et de leurs familles, conformément aux normes de la métropole et aux codes nobiliaires. Aussi Pandoussa Mezeri, en tant que chef de famille, a-t-elle pris soin d'organiser les mariages de ses filles en grande pompe, en fonction de leur rang, afin de répondre, d'une part, aux conventions sociales, et maintenir, de l'autre, le prestige familial, en suivant à la lettre le savoir-faire de l'époque, tout en respectant les traditions du pays.

C'est donc par le biais des mets proposés, que l'on peut reconnaître les influences gastronomiques sur la cuisine crétoise pendant la domination vénitienne. D'une part, le goût épicé et la prééminence carnée dans des plats cuisinés à la façon byzantine, et de l'autre, l'introduction dans le menu de la charcuterie, du riz et des pâtes de la cuisine vénitienne, déjà sous l'influence de Byzance dans une certaine mesure, mais aussi des herbes aromatiques, des légumes et des fruits de la Méditerranée. Pour le service principal, on a déjà mentionné que la denrée de base était carnée : agneaux, levreaux et volaille de toute sorte. Bien que le livre de comptes de Mezeri ne nous permette pas de restituer les recettes, d'autres sources nous renseignent sur les pratiques de 
préparation : rôtis, à la broche ou au pot, d'où les registres concernant les achats de grandes quantités de bois pour ce qu'elle appelle fournaristika [ dépenses du four ", dirait-on. Les viandes étaient accompagnées de légumes et de riz, produit importé et d'un coût très élevé, consommé presque exclusivement par les plus fortunés. Un mets, tout aussi exquis et recherché, était le gibier, offert en abondance lors des festins des noces des familles prospères. Les pigeonneaux et les canetons étaient souvent farcis et cuits au four, tandis que les levreaux, les perdreaux et les grives étaient généralement cuits dans du vin ou du vinaigre, abondamment assaisonnés d'épices (RORATO, 2003), (TUCCI, 1992), (VLASSI, 1998, 64-73).

Le repas était arrosé de deux types de vin, du marouva et du muscadet. D'autres éléments récurrents sont les fromages, les fruits et les confiseries, auxquelles on accorde une attention particulière (VLASSI, 1998, 74). Pendant la période de la Vénétocratie, il était d'usage que l'on offre aux invités de nombreuses friandises, à base d'œufs, de miel ou de sucre et des fruits secs, aux aromates d'eau de rose ou de cannelle, qui annonçaient la douceur et le bonheur de la vie à venir, la fidélité conjugale et les nombreux descendants. D'ailleurs, quel que soit le milieu social ou culturel, le mariage a toujours été associé à des rituels symboliques. Même de nos jours, la surconsommation carnée, le riz, les konfeta, les xerotigana, ne manquent dans aucun banquet de mariage en Crète, et constituent l'une des traditions locales les plus prisées.

\section{Conclusion}

11 Il est incontestable qu'au fil du temps, l'alimentation d'une société vient à la rencontre de sa culture. Temps de guerre et de paix, changements climatiques et brassage interculturel sont des éléments qui influencent l'histoire de la nourriture et contribuent à la formation d'une culture culinaire autour de laquelle fusionnent mets et convives, tout en reflétant attitudes, valeurs, mentalités. La Renaissance, et tout ce qu'elle désigne, joie de vivre, éventail d'empreintes, extraversion, échanges culturels, métissage, ont profondément marqué l'île de Crète à l'ère de sa propre Renaissance, et se sont introduits aussi dans la fête joyeuse du mariage, au moment précis du banquet des noces, où les convives sont en train de faire bonne chère et d'exprimer leurs vœux au nouveau couple, autour d'une grande table d'apparat.

\section{BIBLIOGRAPHIE}

ANAGNOSTAKIS Ilias, 2013, “'The Raw and The Cooked': ways of cooking and serving food in Byzantium", in I. ANAGNOSTAKIS (ed.), Flavours and Delights: Tastes and Pleasures of Ancient and Byzantine Cuisine, Athens: Armos, pp. 175-181.

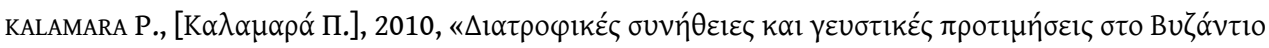

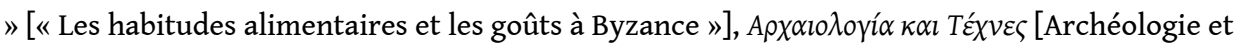
Arts], 116/2, p. 8-17. 
KISLINGER Ewald, 1999, “Christians of the East: Rules and realities of the Byzantine diet”, in FLANDRIN J. L. \& MOLINARI M. (ed.), Food: A Culinary History from Antiquity to the Present, London: Penguin, pp. 194-206.

KODER Johannes, 2005, « L'alimentation quotidienne dans Byzance à base des sources ", in

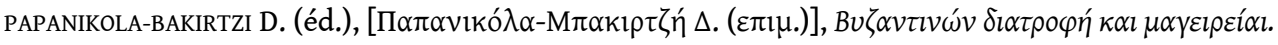

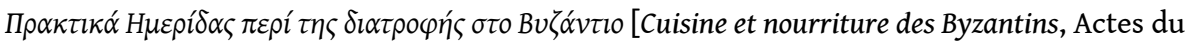
Colloque la Nourriture en Byzance], Athènes, p. 19-26.

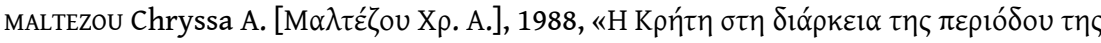

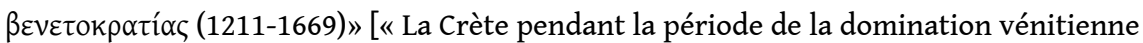

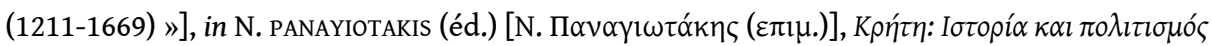

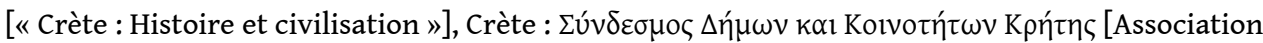
des Villes et Communes de la Crète], vol. 2, p. 107-159.

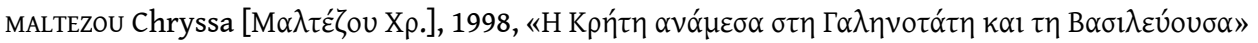
[«Crète entre la Sérénissime et Constantinople »], Cretan Studies 6, pp. 3-20.

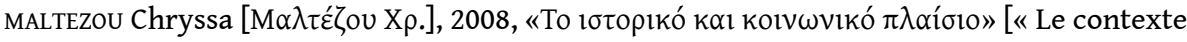

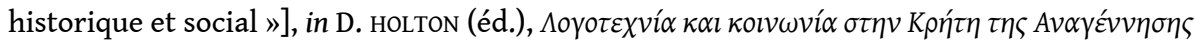
[Littérature et société en Crète pendant la Renaissance], Héraclion, Crète : University Press, p. 21-57.

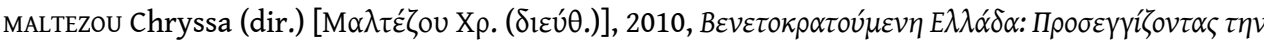

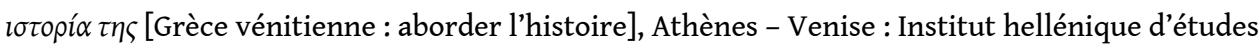
byzantines et post-byzantines, vol. 1, 2 .

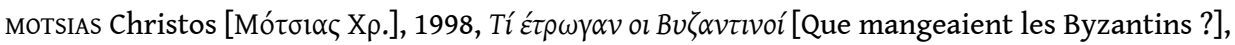
Athènes : Kaktos.

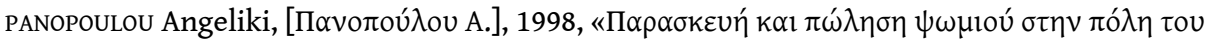
Xóv $\delta \alpha \kappa \alpha\left(16^{\circ \varsigma}-17^{\circ \varsigma} \alpha\right.$ l.) » [ «Préparation et vente de pain dans la ville de Candie (XVI-XVII ${ }^{\mathrm{e}} \mathrm{s}$.) »], in

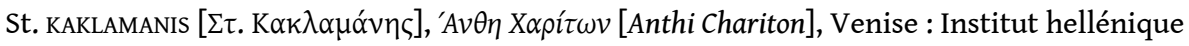
d'études byzantines et post-byzantines, p. 465-495.

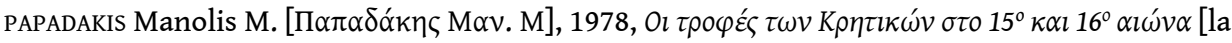
Nourriture des Crétois au XV et $\mathrm{XVI}^{\mathrm{e}}$ siècle], Irakleion Kritis : Platakis.

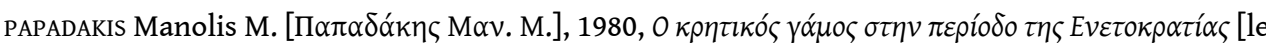

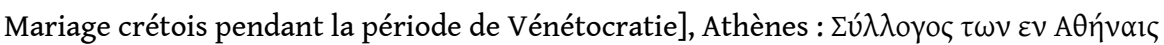

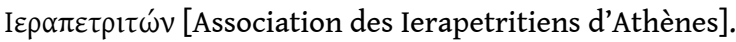

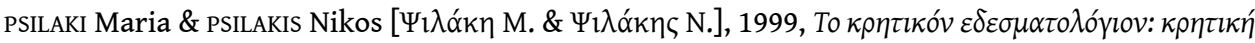
$\pi \alpha \rho \alpha \delta \circ \sigma \iota \alpha \eta \dot{n} \kappa o v \zeta i v \alpha$ [le Menu crétois : cuisine traditionnelle crétoise], Irakleio : Karmanor. PSILAKIS Nikos, 1998, "Aliments des pauvres et les riches dans la Crète de la Vénétocratie », in

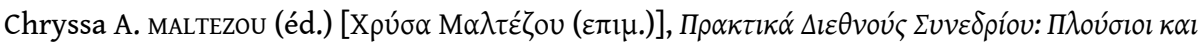

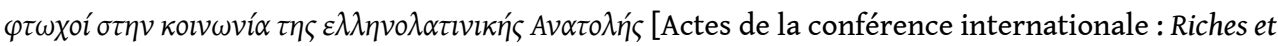
pauvres dans la société de l'Est gréco-latin], Venise : Institut hellénique d'études byzantines et postbyzantines, p. 319-329.

RENAUD Serge, 2004, le Régime crétois, incroyable protecteur de notre santé, Paris : éd. Odile Jacob. TEYSSEYRE Michèle, 1999, Saveurs et senteurs de la Sérénissime, Toulouse : Clairsud. 
TUCCI Hannelore Z., 1992, «Pesca e caccia in laguna» [« Pêche et chasse dans la lagune »], Storia di Venezia [Histoire de Venise], vol. I, Rome, p. 491-514.

RORATO Giampiero, 2003, la Grande Cucina Veneziana: Ricette, storia e cultura della cucina di Venezia [la Grande Cuisine Vénitienne : recettes, histoire et culture de la cuisine de Venise], Vittorio Veneto : Dario De Bastiani.

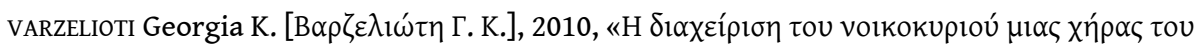

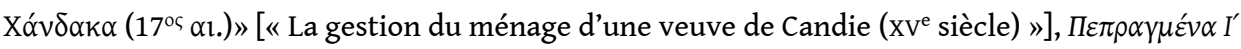

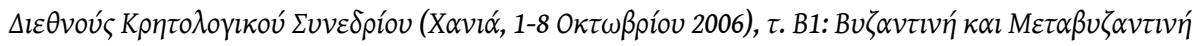

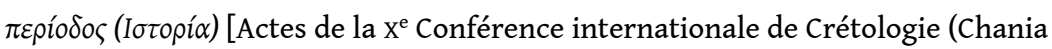

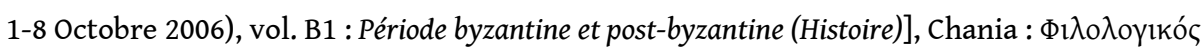

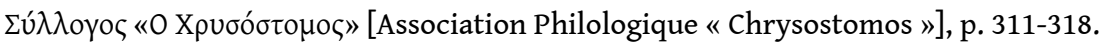

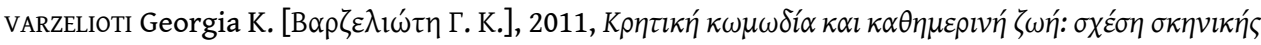

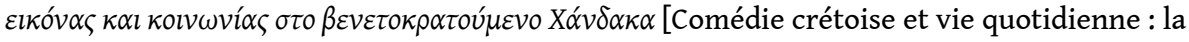
relation entre l'image scénique et la société dans la ville de Candie durant la Vénétocratie], Athènes-Venise : Institut hellénique d'études byzantines et post-byzantines.

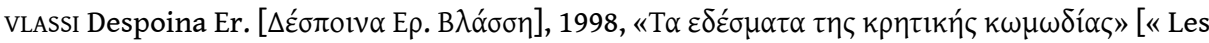

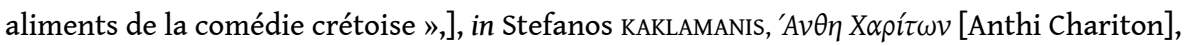
Venise : Institut hellénique d'études byzantines et post-byzantines, p. 56-75.

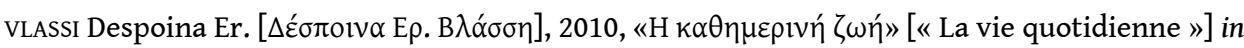

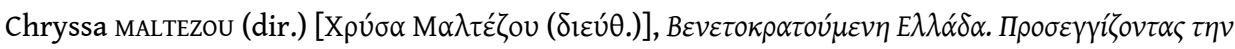

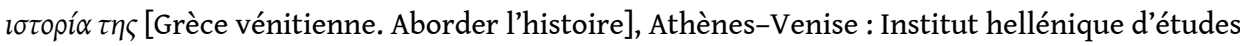
byzantines et post-byzantines, vol. 1, p. 357-387.

\section{NOTES}

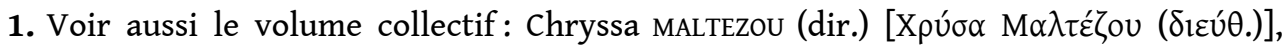

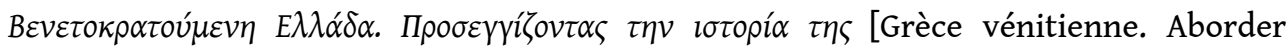
l'histoire], Athènes-Venise, Institut hellénique d'études byzantines et post-byzantines, 2010, vol. 1, 2, avec une riche bibliographie.

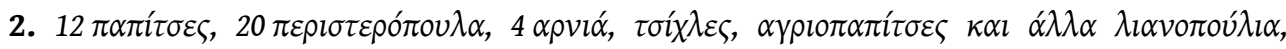

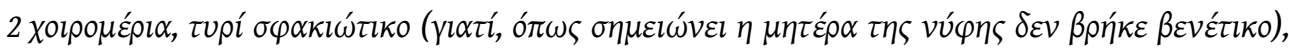

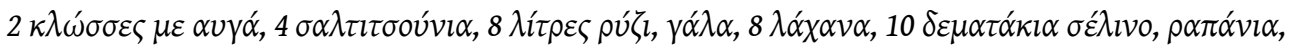

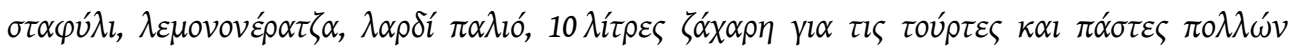

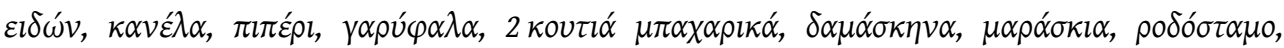

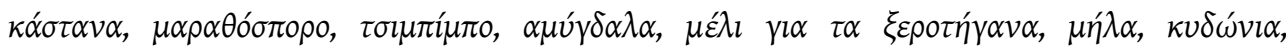

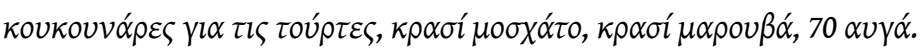




\section{RÉSUMÉS}

En 1609, Pantoussa Mezeri, veuve appartenant à la noblesse de la ville, commence à enregistrer dans un livre de comptes - recouvrant la période 1609-1620 -, les dépenses quotidiennes de son ménage. Pendant les onze années qui suivent, cinq célébrations de mariage ont lieu, celles de ses cinq filles. La veuve Mezeri note de manière détaillée les achats effectués pour chaque table de cérémonie. Le registre en question (à paraître bientôt par l'écrivaine de cet article), représente une source inestimable pour l'histoire culinaire et la culture de table. Il comporte le type, la provenance, la qualité et le coût des denrées, tout en restituant les mœurs de table locales et leur symbolisme dans le contexte social régnant, ainsi que les mentalités et les habitudes de cet environnement multiculturel bourgeois de la capitale du royaume crétois.

In 1609, noblewoman Pandoussa Mezeri, widow of the Cretan physician Victor Mezeris, began to keep records of the daily management of her household. The libro dei conti, written in first person singular, in the form of a journal, is located at the State Archives of Venice and represents a valuable source of information on daily life in Candia during the first half of the 17th century. During the 11 years that followed, the weddings of the five daughters of the Mezeri family took place and Mezeri recorded in great detail the preparations, including shopping for a variety of ingredients, local as well as imported from other areas under Venetian rule and from Venice itself. This "journal", is a unique source of information on the history of food and cuisine culture, in that it describes the kinds, the origins the quality and the prices of foodstuffs, but also the local nutrition traditions and customs with their symbolic meaning in the social context, as well as the mindset and habits marking the multicultural urban civilization in the capital city of the Regno di Candia.

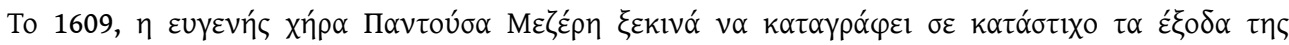

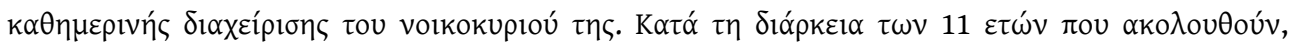

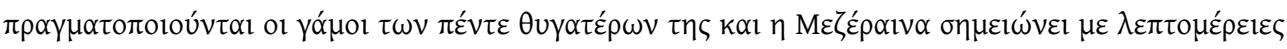

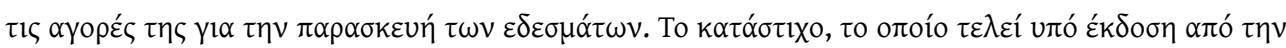

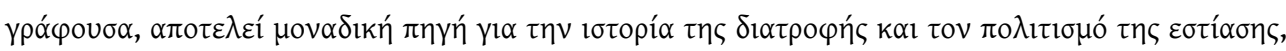
$\pi \varepsilon \rho l \gamma \rho \alpha ́$ ́

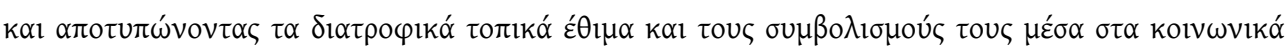

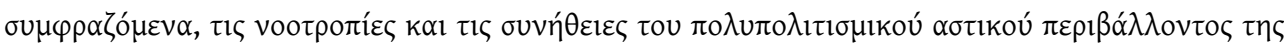

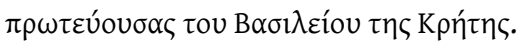


INDEX

motsclestr Yiyecek, Gastronomi, Kültür, Kadınlar, Evlilik, Girit, Rönesans, Venedik işgali (xı11xvı1e), Sosyal tarih, Kültürel tarih

Index chronologique : Renaissance, Vénétocratie (- siècle)

Index géographique : Crète

Mots-clés : nourriture, nourriture, cuisine, cuisine, culture, culture, femme, femme, mariage, mariage

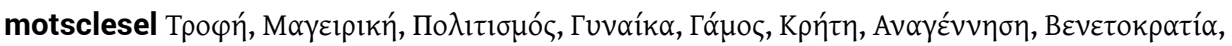

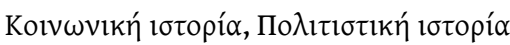

motsclesmk ХРАНА, ГАСТРОНОМИЈА, КУЛТУРА, ЖЕНИТЕ, БРАКОТ, КРИТ, РЕНЕСАНСАТА, ВЕНЕЦИЈАНСКИ ОКУПАЦИЈА (xiii-Xvii), СОЦИЈАЛНА ИСТОРИЈА, КУЛТУРНА ИСТОРИЈА

Keywords : Food, Cooking, Culture, Woman, Marriage, Crete, Renaissance, Venetocracy (13-17th centuries), Social history, Cultural history

Thèmes: Histoire sociale, Histoire culturelle

\section{AUTEUR}

GEORGIA K. VARZELIOTI

Université Nationale et Capodistrienne d'Athènes 